\title{
Endothelial Arginase: A New Target in Atherosclerosis
}

\author{
Zhihong Yang, MD, and Xiu-Fen Ming, $M D, P h D$
}

\author{
Corresponding author \\ Zhihong Yang, MD \\ Vascular Biology, Department of Medicine, Division of \\ Physiology, University of Fribourg, Rue du Musée 5, CH-1700 \\ Fribourg, Switzerland. \\ E-mail: zhihong.yang@unifr.ch
}

Decreased endothelial nitric oxide (NO) bioavailability as it relates to endothelial dysfunction plays an important role in various cardiovascular disorders, including atherosclerosis. Recent research has provided evidence that endothelial dysfunction in atherosclerosis are not primarily caused by decreased endothelial NO synthase (eNOS) gene expression, but rather deregulation of eNOS enzymatic activity, which contributes to the increased oxidative stress in atherosclerosis. Among other mechanisms, the substrate L-arginine is an important limiting factor for NO production. Emerging evidence demonstrates that L-arginine is not only converted to $\mathrm{NO}$ via eNOS, but also metabolized to urea and L-ornithine via arginase in endothelial cells. Hence, arginase competes with eNOS for the substrate L-arginine, resulting in deceased NO production. There are an increasing number of studies showing that enhanced arginase gene expression and/or activity contribute to endothelial dysfunction in various cardiovascular disorders, including atherosclerosis. Thus, endothelial arginase may represent a new therapeutic target in atherosclerosis.

\section{Introduction}

Atherosclerotic coronary artery disease remains the leading cause of death in industrialized countries, despite the remarkable progress being made toward understanding the mechanisms and therapeutic modalities in recent decades [1].
Pathogenesis of atherosclerosis is a complex process involving vasoconstriction, intimal thickening, and thrombus formation [1]. Dysfunctions of numerous cell types in the vascular wall (ie, endothelial and smooth muscle cells) and circulating blood (ie, platelets and white blood cells) all contribute to the disease process [1]. Research in recent decades provides substantial evidence that endothelial dysfunction as it relates to decreased bioavailability of endothelial nitric oxide (NO) plays an important role in atherogenesis [2,3].

Endothelium-derived NO is produced from the substrate L-arginine via endothelial NO synthase (eNOS), promotes vasodilation, and inhibits inflammation, platelet aggregation, and vascular smooth muscle cell (SMC) proliferation [4]. It is, therefore, not surprising that loss of endothelial NO function initiates and accelerates atherogenesis, as demonstrated by various experiments with animal models [5,6]. Although our understanding of biochemical regulation of endothelial NO bioactivity has remarkably advanced on various levels of eNOS gene expression - eNOS enzymatic activity to degradation of NO [7] — no single mechanism can fully explain endothelial dysfunction in atherosclerosis. Initial studies suggest that endothelial dysfunction in atherosclerosis is due to a decrease in eNOS gene expression [8]. Research in more recent years, however, provides evidence suggesting that reduced NO bioavailability, mainly due to a decrease in eNOS enzymatic activity rather than eNOS gene expression and accelerated inactivation of NO by oxidative stress, is the central mechanism of endothelial dysfunction in atherosclerosis [9]. This conclusion is supported by most studies of atherosclerotic animal models, showing an unchanged or even augmented protein level of eNOS in atherosclerotic arteries, despite the presence of endothelial dysfunction [10-12,13••]. Moreover, studies with human aortic and coronary arterial tissues obtained from autopsy or from transplant donors found only a significantly deceased eNOS gene expression in endothelial cells in advanced but not early atherosclerotic lesions [14,15]. Most recently, a study with human coronary atherectomy specimens showed a higher eNOS gene expression in patients with acute coronary syndromes than those with stable angina [16]. It is of particular importance to note that Ozaki et al. [17] recently showed acceleration of atherosclerotic lesion formation 
in $\mathrm{ApoE}^{-/-}$mice overexpressing bovine eNOS transgene. Controversial results were, however, reported by van Haperen et al. [18] with the same experimental approach. The discrepancies in the results of the two studies are not clear. It has been speculated that the difference in severity of hypercholesterolemia and/or changes in blood pressure in animals between the two studies may explain the controversial results. Based on the outcomes of these, it can be asserted that endothelial dysfunction in atherosclerosis is not primarily caused by decreased eNOS gene expression. The studies also implicate that eNOS gene transfer into atherosclerotic arteries, which was initially proposed as a therapeutic approach to treat atherosclerosis, may not be generalized and should be carefully revisited. It is emerging that functional change in eNOS enzymatic activity rather than eNOS gene suppression plays an important role in endothelial dysfunction in atherosclerosis.

\section{Controversy of L-Arginine Supplemental Therapy in Atherosclerosis}

The enzymatic activity of eNOS is affected by multiple factors, including post-translational modification associated with subcellular localization; the interacting proteins such as caveolin-1 and heat shock protein 90; and phosphorylation/dephosphorylation by protein kinases stimulated by hormonal agonists [7]. In addition, increase in endogenous eNOS inhibitor asymmetric dimethylarginine (ADMA), deficiency in co-factor tetrahydrobiopterin $\left(\mathrm{BH}_{4}\right)$, and deficiency in the substrate L-arginine have been proposed to cause so-called "uncoupling of eNOS" in atherosclerosis, a situation in which eNOS produces free radical superoxide anion instead of NO $[17,19]$. This mechanism may further contribute to inactivation of bioactive $\mathrm{NO}$ in atherosclerosis mediated by oxidative stress mediated by other enzymes such as NADPHoxidase [19].

The bioavailability of L-arginine as a limiting factor for endothelial NO production was first recognized in the early 1990s. Several groups demonstrated that acute and chronic supplementation of L-arginine improves endothelial vasodilator responses in cholesterol fed animals and in patients with hypercholesterolemia and atherosclerosis [20-23]. Following these early studies, numerous studies with supplemental Larginine therapy have been conducted in animal models and in humans; however, no consistent results can be achieved [24••]. In recent years, there has been an increasing number of studies showing either no effect or no sustained effects on endothelial function by L-arginine supplementation [6,25-30]. Harmful effects on atherosclerotic lesion formation in ApoE/iNOS double-knockout mice have been reported [31]. In this mouse model, the beneficial effect mediated by iNOS knockout on atherosclerotic lesion formation is eliminated with chronic Larginine supplementation [31]. In line with this report, an increase in superoxide anion production was also demonstrated in atherosclerotic rabbit aortas treated with L-arginine [32]. The reason for the inconsistent results with L-arginine supplementation therapy is not clear. It might be due to the complex of the atherosclerotic disease process as well as the complex of biochemical metabolic pathways of the semi-amino acid L-arginine [33].

\section{Multiple Metabolic Pathways of L-Arginine}

L-arginine, depending on cell types and tissues, undergoes multiple biochemical metabolisms and serves as precursor for a number of biologically active compounds involved in regulation of various cellular functions [33] (Fig. 1). As mentioned earlier, L-arginine is oxidized to NO and L-citrulline via eNOS in endothelial cells. Additionally, it is used for protein synthesis via arginyl t-RNA synthetase. L-arginine is also used for production of creatine via arginine:glycine amidinotransferase and guanidinoacetate $\mathrm{N}$-methyltransferase, a pathway more restricted to the kidney and pancreas. Furthermore, L-arginine can be converted to L-agmatine via arginine decarboxylase (ADC) and to L-ornithine and urea via arginase, which is part of the urea cycle. Urea is then excreted through the kidney (Fig. 1). L-ornithine is further metabolized via ornithine decarboxylase (ODC) into polyamines that are important for cell proliferation [33]. L-ornithine can also be metabolized via ornithine aminotransferase (OAT) to L-proline, which is used for collagen production and deposition [33]. Hence, the production of polyamines and L-proline from L-arginine/Lornithine may play a role in negative vascular remodeling [34] (Fig. 1). Among the metabolisms, there is recently increasing evidence suggesting a potential role of arginase in regulation of endothelial NO production by competing with eNOS for the substrate L-arginine. Increase in arginase activity has recently been demonstrated to play an important role in endothelial dysfunction under several pathophysiologic conditions, including atherosclerosis (see later).

\section{Role of Arginase in Endothelial Dysfunction}

In mammals, there are two types of arginase, arginase I and II which are encoded by different genes [35]. Arginase I is a cytosolic enzyme, expressed most abundantly in the liver. The primary function of arginase $\mathrm{I}$ is ammonia detoxification. Arginase II is a mitochondrial enzyme, expressed primarily in the kidney or other extrahepatic tissues, including blood vessels [36]. The primary function of this type of enzyme is biosynthesis of polyamines and amino acids ornithine, proline, and glutamate [36]. Vascular endothelial and smooth muscle cells express both arginase I and II, but the relative level of the two types of enzyme seems different depending on species [13••,37-39]. In human endothelial cells, arginase II seems to be the predominant isoenzyme $[13 \bullet \bullet, 39]$. The expression and activity of arginase II were found to be increased in human diabetic corpus cavernosum, and inhibition of the enzyme enhances NO-dependent relaxation of corpus cavernosum smooth muscle [40,41], suggesting a potential role for arginase II in negative regulation of NO production in diabetic erectile dysfunction. Similarly, arginase activity was also found to be higher in the aortas of type 1 and type 2 diabetic animal models 
(Yang and Ming, Unpublished results). Our recent study demonstrates that in atherosclerotic aortas of $\mathrm{ApoE}^{-/-}$mice, arginase II was abundantly expressed, whereas arginase I was not detectable [13••]. Although the expression of arginase II was comparable between atherosclerotic and wild-type mice, the activity of the enzyme was increased in $\mathrm{ApoE}^{-/-}$animals, suggesting that increased enzymatic activity of arginase II plays a predominant role in atherosclerotic endothelial dysfunction.

It is quite surprising that L-arginine causes vasoconstriction in isolated mouse aortas, which is in contrast to the observation in rats and humans in which L-arginine evokes vascular relaxation by producing NO [13••]. Most interestingly, the contraction induced by L-arginine is much more pronounced in atherosclerotic ApoE ${ }^{-/-}$mice than in control animals. This contraction induced by L-arginine can be converted to a greater relaxation by the arginase inhibitor Lnorvaline in atherosclerotic ApoE $\mathrm{E}^{-/-}$mice than that in wild-type animals [13••]. The results demonstrate a dominant role of increased arginase activity in atherosclerotic endothelial dysfunction. The results also imply that in mouse aortas, particularly in the atherosclerotic $\mathrm{ApoE}^{-/-}$aortas, and perhaps also in humans depending on the stage of atherosclerosis, Larginine can be metabolized by arginase to certain vasoconstrictive intermediate products. This hypothesis, if proven true, may explain the controversial results with Larginine supplementation therapy. In line with the greater vascular relaxation induced by L-arginine in the presence of the arginase inhibitor, eNOS protein levels are also higher in atherosclerotic $\mathrm{ApoE}^{-/-}$aortas than in the wild-type animals [13••]. The results further support the concept that endothelial dysfunction in atherosclerosis is mainly due to decreased NO bioavailability rather than gene expression. A simple administration of L-arginine alone as anti-atherosclerotic therapy should not be generalized. Future research designed to target arginase specifically in the vasculature may provide a novel therapeutic approach to treat atherosclerosis and perhaps also other cardiovascular disorders.

Indeed, the role of arginase in endothelial dysfunction has been recently extended to other pathophysiologic conditions associated with vascular disorders in animal models such as aging [42,43], ischemia-reperfusion-induced endothelial dysfunction [44], and various types of hypertension $[45,46,47 \cdot \bullet]$. Most recently, the role of arginase in endothelial dysfunction has also been demonstrated in patients with primary pulmonary hypertension [48] and in patients with sickle cell disease [49••]. Importantly, arginase activity in blood plasma or red blood cells was found to be increased in patients with sickle cell disease, and is independently associated with pulmonary hypertension and mortality in these patients [49••].

\section{Regulation of Arginase Gene Expression and Activity in Endothelial Cells}

Although there is evidence that arginase gene expression and/or activity is increased in various cardiovascular disorders, little information is available on the underlying regulatory

mechanisms in the vasculature under the disease conditions, including atherosclerosis. Inflammatory cytokines have been shown to induce gene expression of arginase I and II in bovine endothelial cells, which is most likely mediated through transactivation of epidermal growth factor (EGF) receptors by the cytokines [50]. Our recent study provided the first evidence for the important role of RhoA/ROCK pathway in upregulation of arginase activity in human endothelial cells and in atherosclerotic blood vessels [13••]. We found that in human endothelial cells and atherosclerotic aortas of $\mathrm{ApoE}^{-/-}$mice, arginase I is not detectable by immunoblotting, whereas arginase II is abundantly expressed. Although the expression of arginase II is comparable in atherosclerotic and wild-type mice, the activity of the enzyme is significantly increased in $\mathrm{ApoE}^{-/-}$ animals, suggesting that increased enzymatic activity of arginase II plays a predominant role in atherosclerotic endothelial dysfunction. The higher arginase activity in the atherosclerotic aorta is associated with higher RhoA protein level, suggesting a role for RhoA in upregulation of arginase activity. Indeed, in cultured human endothelial cells, arginase II enzymatic activity, but not protein expression, is enhanced by thrombin, a potent activator of RhoA/ROCK in endothelial cells, after 18 to 24 hours of stimulation. The enhanced arginase activity can be prevented by inhibitors of the RhoA/ROCK pathway. In line with this observation, adenovirus-mediated ectopic expression of a constitutively active mutant of RhoA or ROCK significantly enhanced arginase activity but not the gene expression in the cells. The results suggest that the Rho/ROCK pathway stimulates arginase II via regulating the enzymatic activity rather than gene expression. This regulatory model of arginase II in human endothelial cells is further supported by a study by Bachetti et al. [39], who showed that arginase activity but not gene expression in the cells was increased after 24 hours of stimulation with inflammatory cytokine mixture. The exact regulatory mechanisms of arginase activity in the cells and in atherosclerotic blood vessels remain an interesting topic for future research.

\section{Conclusions}

Endothelial dysfunction in atherosclerosis is primarily caused by functional changes in eNOS enzymatic activity. Among other mechanisms, vascular endothelial arginase is emerging to play a substantial role in endothelial dysfunction under various pathophysiologic conditions, including atherosclerosis. Enhanced endothelial arginase activity competes with eNOS for the substrate L-arginine, resulting in decreased NO production. Various studies showed that inhibition of vascular arginase activity improves endothelial dysfunction under various pathologic conditions. These results suggest that targeting endothelial arginase may represent a new therapeutic approach for treatment of atherosclerosis and other cardiovascular disorders.

\section{Acknowledgments}


The original work of this group was supported by the Swiss National Science Foundation (Nr 31-63811.00 and 3100A0105917) and Swiss Heart Foundation.

\section{References and Recommended Reading}

Papers of particular interest, published recently,

have been highlighted as:

- Of importance

•• Of major importance

1. Libby P, Theroux P: Pathophysiology of coronary artery disease. Circulation 2005, 111:3481-3488.

2. Halcox JP, Schenke WH, Zalos G, et al.: Prognostic value of coronary vascular endothelial dysfunction. Circulation 2002, 106:653-658.

3. Bugiardini R, Manfrini O, Pizzi C, et al.: Endothelial function predicts future development of coronary artery disease: a study of women with chest pain and normal coronary angiograms. Circulation 2004, 109:2518-2523.

4. Yang Z, Luscher TF: Vascular endothelium. In PanVascular Medicine. Edited by Lanzer P, Topol EJ. Berlin-Heidelberg-New York: Springer; 2002:190-204.

5. Knowles JW, Reddick RL, Jennette JC, et al.: Enhanced atherosclerosis and kidney dysfunction in eNOS(-/-)Apoe(-/-) mice are ameliorated by enalapril treatment. $J$ Clin Invest 2000 , 105:451-458.

6. Kauser $K$, da Cunha V, Fitch $R$, et al.: Role of endogenous nitric oxide in progression of atherosclerosis in apolipoprotein $\mathrm{E}$ deficient mice. Am J Physiol Heart Circ Physiol 2000, 278:H1679H1685.

7. Sessa WC: eNOS at a glance. J Cell Sci 2004, 117:2427-2429.

8. Oemar BS, Tschudi MR, Godoy N, et al.: Reduced endothelial nitric oxide synthase expression and production in human atherosclerosis. Circulation 1998, 97:2494-2498.

9. Davignon J, Ganz P: Role of endothelial dysfunction in atherosclerosis. Circulation 2004, 109(23 Suppl 1):III27-III32.

10. Matsumoto T, d'Uscio LV, Eguchi D, et al.: Protective effect of chronic vitamin $C$ treatment on endothelial function of apolipoprotein E-deficient mouse carotid artery. J Pharmacol Exp Ther 2003, 306:103-108.

11. Godecke A, Ziegler M, Ding Z, et al.: Endothelial dysfunction of coronary resistance vessels in apoE-/- mice involves NO but not prostacyclin-dependent mechanisms. Cardiovasc Res 2002, 53:253-262.

12. d'Uscio LV, Baker TA, Mantilla CB, et al.: Mechanism of endothelial dysfunction in apolipoprotein E-deficient mice. Arterioscler Thromb Vasc Biol 2001, 21:1017-1022.

13.• Ming XF, Barandier C, Viswambharan H, et al.: Thrombin stimulates human endothelial arginase enzymatic activity via RhoA/ROCK pathway: implications for atherosclerotic endothelial dysfunction. Circulation 2004, 110:3708-3714.

This is the first study showing that endothelial arginase activity is regulated by Rho/ROCK pathway. Arginase activity is increased in atherosclerotic aortas in ApoE-/mice and plays a substantial role in endothelial dysfunction in atherosclerosis.

14. Wilcox JN, Subramanian RR, Sundell CL, et al.: Expression of multiple isoforms of nitric oxide synthase in normal and atherosclerotic vessels. Arterioscler Thromb Vasc Biol 1997, 17:2479-2488.

15. Fukuchi M, Giaid A: Endothelial expression of endothelial nitric oxide synthase and endothelin-1 in human coronary artery disease. Specific reference to underlying lesion. Lab Invest 1999, 79:659-670.

16. Rossi ML, Marziliano N, Merlini PA, et al.: Phenotype commitment in vascular smooth muscle cells derived from coronary atherosclerotic plaques: differential gene expression of endothelial nitric oxide synthase. Eur J Histochem 2005, 49:39-46.

17. Ozaki M, Kawashima S, Yamashita T, et al.: Overexpression of endothelial nitric oxide synthase accelerates atherosclerotic lesion formation in apoE-deficient mice. $J$ Clin Invest 2002, 110:331-340.

18. van Haperen R, de Waard M, van Deel E, et al.: Reduction of blood pressure, plasma cholesterol, and atherosclerosis by elevated endothelial nitric oxide. $J$ Biol Chem 2002, 277:48803-48807.

19. Munzel T, Daiber A, Ullrich V, et al.: Vascular consequences of endothelial nitric oxide synthase uncoupling for the activity and expression of the soluble guanylyl cyclase and the cGMPdependent protein kinase. Arterioscler Thromb Vasc Biol 2005, 25:1551-1557.

20. Drexler H, Zeiher AM, Meinzer K, et al.: Correction of endothelial dysfunction in coronary microcirculation of hypercholesterolaemic patients by L-arginine. Lancet 1991, 338:1546-1550.

21. Cooke JP, Singer AH, Tsao P, et al.: Antiatherogenic effects of $\mathbf{L}$ arginine in the hypercholesterolemic rabbit. J Clin Invest 1992, 90:1168-1172.

22. Creager MA, Gallagher SJ, Girerd XJ, et al.: L-arginine improves endothelium-dependent vasodilation in hypercholesterolemic humans. J Clin Invest 1992, 90:1248-1253.

23. Dubois-Rande JL, Zelinsky R, Roudot F, et al.: Effects of infusion of L-arginine into the left anterior descending coronary artery on acetylcholine-induced vasoconstriction of human atheromatous coronary arteries. Am J Cardiol 1992, 70:1269-1275.

24.•• Loscalzo J: Adverse effects of supplemental L-arginine in atherosclerosis: consequences of methylation stress in a complex catabolism? Arterioscler Thromb Vasc Biol 2003, 23:3-5.

A critical editorial comment analyzing controversial results with L-arginine supplemental therapy obtained from experimental and clinical studies.

25. Jeremy RW, McCarron H, Sullivan D: Effects of dietary L-arginine on atherosclerosis and endothelium-dependent vasodilatation in the hypercholesterolemic rabbit. Response according to treatment duration, anatomic site, and sex. Circulation 1996, 94:498-506.

26. Oomen CM, van Erk MJ, Feskens EJ, et al.: Arginine intake and risk of coronary heart disease mortality in elderly men. Arterioscler Thromb Vasc Biol 2000, 20:2134-2139.

27. Blum A, Hathaway L, Mincemoyer R, et al.: Oral L-arginine in patients with coronary artery disease on medical management. Circulation 2000, 101:2160-2164.

28. Walker HA, McGing E, Fisher I, et al.: Endothelium-dependent vasodilation is independent of the plasma L-arginine/ADMA ratio in men with stable angina: lack of effect of oral $L$-arginine on endothelial function, oxidative stress and exercise performance. J Am Coll Cardiol 2001, 38:499-505.

29. Wennmalm A, Edlund A, Granstrom EF, et al.: Acute supplementation with the nitric oxide precursor L-arginine does not improve cardiovascular performance in patients with hypercholesterolemia. Atherosclerosis 1995, 118:223-231.

30. Miller HI, Dascalu A, Rassin TA, et al.: Effects of an acute dose of L-arginine during coronary angiography in patients with chronic renal failure: a randomized, parallel, double-blind clinical trial. Am J Nephrol 2003, 23:91-95.

31. Chen J, Kuhlencordt P, Urano F, et al.: Effects of chronic treatment with $\mathrm{L}$-arginine on atherosclerosis in apoE knockout and apoE/inducible NO synthase double-knockout mice. Arterioscler Thromb Vasc Biol 2003, 23:97-103.

32. Simonet S, Rupin A, Badier-Commander C, et al.: Evidence for superoxide anion generation in aortas of cholesterol-fed rabbits treated with L-arginine. Eur J Pharmacol 2004, 492:211-216.

33. Wu G, Morris SM Jr: Arginine metabolism: nitric oxide and beyond. Biochem J 1998, 336:1-17.

34. Durante W, Liao L, Reyna SV, et al.: Transforming growth factorbeta(1) stimulates $L$-arginine transport and metabolism in vascular smooth muscle cells: role in polyamine and collagen synthesis. Circulation 2001, 103:1121-1127. 
35. Morris SM Jr, Bhamidipati D, Kepka-Lenhart D: Human type II arginase: sequence analysis and tissue-specific expression. Gene 1997, 193:157-161.

36. Cederbaum SD, Yu H, Grody WW, et al.: Arginases I and II: Do their functions overlap? Mol Genet Metab 2004, 81(Suppl 1):S38S44.

37. Zhang C, Hein TW, Wang W, et al.: Constitutive expression of arginase in microvascular endothelial cells counteracts nitric oxide-mediated vasodilatory function. FASEB J 2001, 15:12641266.

38. Buga GM, Singh R, Pervin S, et al.: Arginase activity in endothelial cells: inhibition by NG-hydroxy-L-arginine during high-output NO production. Am J Physiol 1996, 271:H1988-H1998.

39. Bachetti T, Comini L, Francolini G, et al.: Arginase pathway in human endothelial cells in pathophysiological conditions. $J \mathrm{Mol}$ Cell Cardiol 2004, 37:515-523.

40. Bivalacqua TJ, Hellstrom WJ, Kadowitz PJ, et al.: Increased expression of arginase II in human diabetic corpus cavernosum: in diabetic-associated erectile dysfunction. Biochem Biophys Res Commun 2001, 283:923-927.

41. Cama E, Colleluori DM, Emig FA, et al.: Human arginase II: crystal structure and physiological role in male and female sexual arousal. Biochemistry 2003, 42:8445-8451.

42. Berkowitz DE, White R, Li D, et al.: Arginase reciprocally regulates nitric oxide synthase activity and contributes to endothelial dysfunction in aging blood vessels. Circulation 2003, 108:2000-2006.

43. Sakai Y, Masuda H, Kihara K, et al.: Involvement of increased arginase activity in impaired cavernous relaxation with aging in the rabbit. $J$ Urol 2004, 172:369-373.

44. Hein TW, Zhang C, Wang W, et al.: Ischemia-reperfusion selectively impairs nitric oxide-mediated dilation in coronary arterioles: counteracting role of arginase. FASEB $J$ 2003, 17:2328-2330.

45. Zhang C, Hein TW, Wang W, et al.: Upregulation of vascular arginase in hypertension decreases nitric oxide-mediated dilation of coronary arterioles. Hypertension 2004, 44:935-943.

46. Johnson FK, Johnson RA, Peyton KJ, et al.: Arginase inhibition restores arteriolar endothelial function in Dahl rats with saltinduced hypertension. Am J Physiol Regul Integr Comp Physiol 2004, Dec 9, [Epub ahead of print].

47.• Demougeot C, Prigent-Tessier A, Marie C, et al.: Arginase inhibition reduces endothelial dysfunction and blood pressure rising in spontaneously hypertensive rats. J Hypertens 2005, 23:971-978.

An important study demonstrating that arginase expression and activity are increased in spontaneous hypertensive rats, and treatment of the rat with an arginase inhibitor improves endothelial function and decreases blood pressure in the animals.

48. $\mathrm{Xu} \mathrm{W}$, Kaneko FT, Zheng S, et al.: Increased arginase II and decreased NO synthesis in endothelial cells of patients with pulmonary arterial hypertension. FASEB J 2004, 18:1746-1748.

49.• Morris CR, Kato GJ, Poljakovic M, et al.: Dysregulated arginine metabolism, hemolysis-associated pulmonary hypertension, and mortality in sickle cell disease. JAMA 2005, 294:81-90.

The first clinical study showing that increased arginase activity in plasma and red blood cells is independently associated with pulmonary hypertension and mortality in patients with sickle cell disease.

50. Nelin LD, Chicoine LG, Reber KM, et al.: Cytokine-induced endothelial arginase expression is dependent on epidermal growth factor receptor. Am J Respir Cell Mol Biol 2005, Jun 30, [Epub ahead of print]. 
Figure 1. The multiple metabolic pathways of L-arginine. ADCarginine decarboxylase; eNOS-endothelial nitric oxide synthase; NO-nitric oxide; OAT - ornithine aminotransferase; ODC-ornithine decarboxylase; SMC-smooth muscle cell.

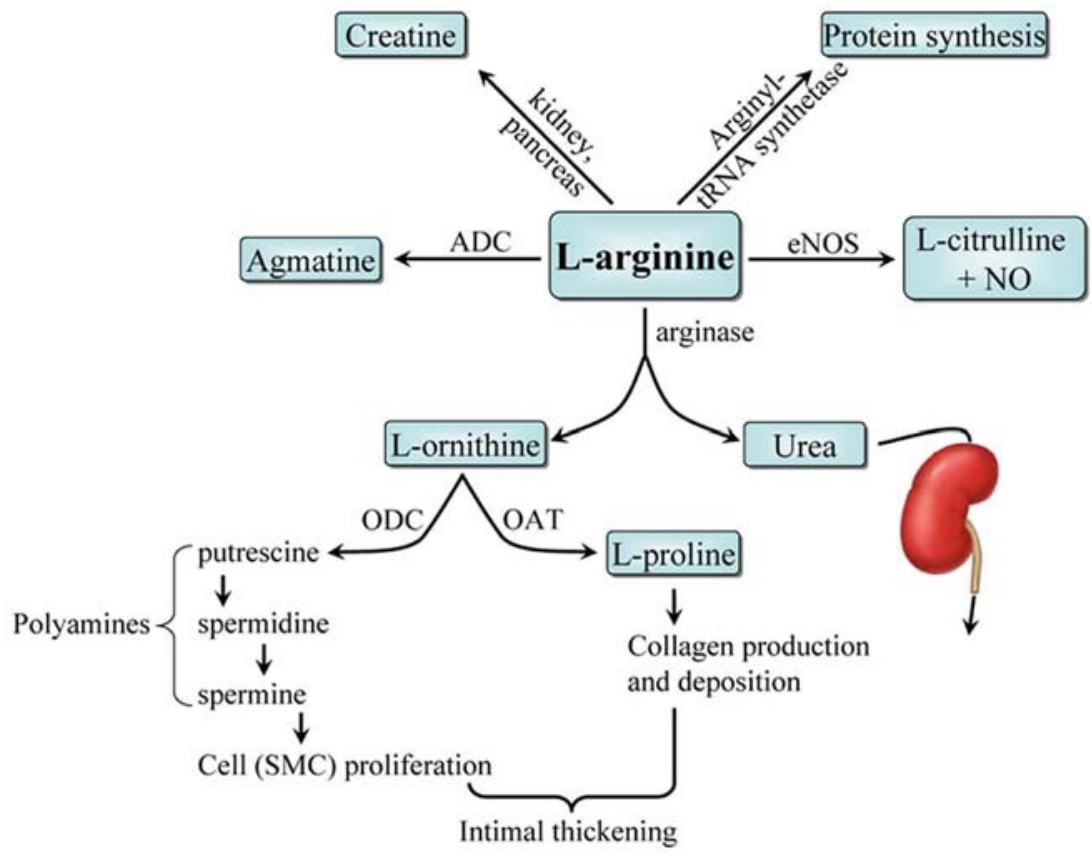

